\section{Taking PET for a Walk - an Unusual Cause of Bilateral Leg Claudication}

\section{To the Editor:}

Large-vessel giant cell arteritis (LV-GCA) is a rare condition that is increasingly recognized with the advent of imaging techniques such as positron emission tomography (PET). However, the utility of PET in extracranial GCA is poorly defined and rarely reported, especially in lower-limb LV-GCA. We describe a case of GCA affecting the lower limbs in which PET, corroborated with computed tomography angiogram (CTA) and duplex ultrasound, was used to accurately assess and monitor the activity of this rare arteritis.

A 59-year-old woman with minimal atherosclerotic risk factors presented with a 3-month history of progressive bilateral leg claudication. She initially noticed tightness in her right calf after walking 100 meters on level ground. The discomfort was proportional to exertion, and would promptly resolve with rest. There was similar, although less intense, discomfort in her left calf, and associated bilateral numbness in her middle 3 toes. Three months previously she had unlimited exercise tolerance on level ground. One month after the onset of claudication, her symptoms progressed to include bilateral thigh discomfort on exertion. There was no scalp tenderness, visual disturbance, jaw or tongue claudication, or polymyalgic or joint symptoms. She had lost 2.5 kilograms ( $4 \%$ total body weight) over 3 months.

Her medical history was unremarkable. Her atherosclerotic risk factors included a brief 2.5 pack-year smoking history and a family history of lower-limb arterial disease (sister requiring femoropopliteal bypass at age 49 years) and old-age ischemic heart disease.

On examination, the left popliteal artery and bilateral pedal pulses were absent. The right popliteal pulse was weakly palpable, and femoral pulses were present. There were no femoral, renal, or lower abdominal bruits. Temporal arteries were nontender, with normal pulses. There were no carotid, subclavian, or axillary artery bruits. Lower-limb neurological examination was normal.

Initial blood tests showed a mildly elevated C-reactive protein at 9.2 $\mathrm{mg} / \mathrm{l}$ (normal $0-5 \mathrm{mg} / \mathrm{l}$ ), with normal erythrocyte sedimentation rate and full blood count. Hepatitis B and C serology, cryoglobulins, autoimmune and vasculitic markers, thrombophilia screen (protein C \& S, homocysteine, Factor V Leiden, prothrombin gene G20210A polymorphism), and antiphospholipid antibodies were negative. The lipid profile, random blood glucose, creatine kinase, creatinine clearance, and urinalysis were also unremarkable.

Initial duplex ultrasound examination of lower-limb arteries revealed significant stenotic femorotibial disease bilaterally, suspicious for vasculitis. There was diffuse and irregular circumferential intima-media thickening of the arterial wall extending from the origins of both superficial femoral arteries to the popliteal and calf arteries, with complete occlusion of the right tibioperoneal trunk. Specifically, along the length of the left superficial femoral artery, there were $1 \mathrm{~cm}$-length focal stenoses of $50 \%-75 \%$ diameter reduction at 12,15 , and $18 \mathrm{~cm}$ below the groin crease, and associated periadventicial, hypoechogenic lucencies. The right superficial femoral artery had marked, irregularly thickened walls from its origin along its full length. Minor luminal irregularities were also present. Two focal short segments of $50 \%$ and $75 \%$ diameter stenoses were present at 5 and $13 \mathrm{~cm}$ below the right groin crease. No calcifications were visualized in the lower-limb arterial tree.

CTA of the aorta and lower limbs showed smooth, circumferential wall thickening highly suggestive of arteritis, involving the abdominal aorta, bilateral common femoral, superficial femoral, profunda femoris arteries, and their thigh and calf branches.

Whole-body PET showed an increased tracer uptake pattern suggestive of large-vessel vasculitis, with distribution corresponding to the duplex and CTA findings (Figure 1). Duplex ultrasound of the temporal arteries showed occlusion of the distal left temporal artery with echolucent intimamedia thickening suspicious of arteritis. GCA was confirmed on left tem-

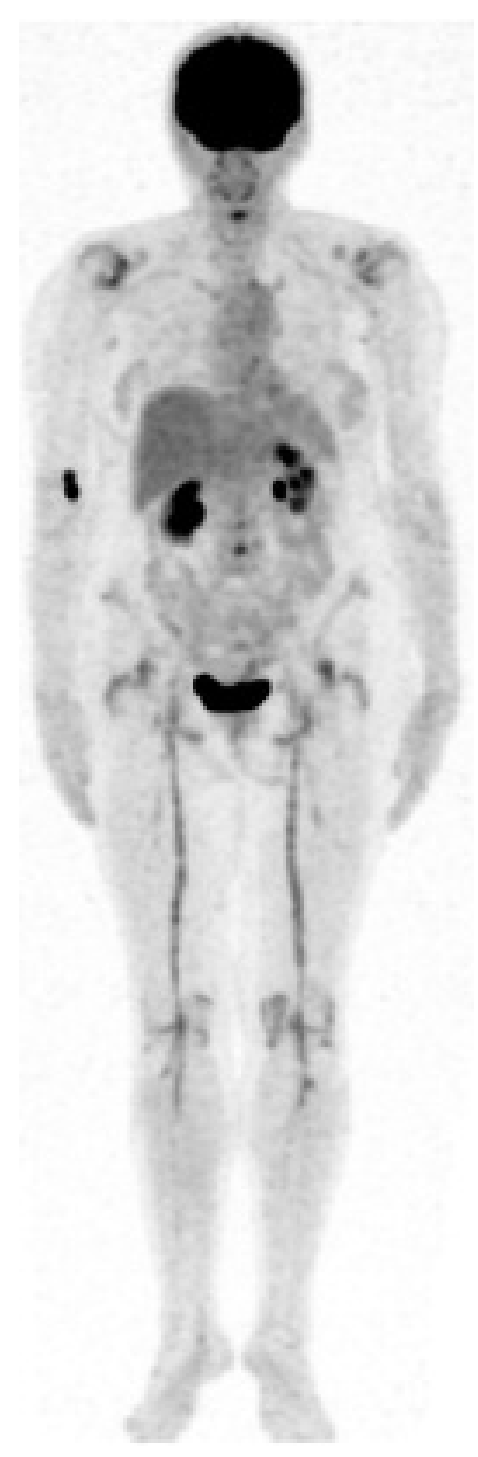

Figure 1. Baseline frontal PET scan showing mild to moderate diffusely increased uptake throughout the bilateral femoral and popliteal arteries, with possible extension to the external iliac arteries, suggestive of a largevessel vasculitis pattern. There is also mild, subclinical increased uptake in the left subclavian artery.

poral artery biopsy, with focal lymphocytic inflammatory infiltrate, scattered giant cells, and mononuclear histocytes visualized within the media.

The patient was treated initially with prednisone $60 \mathrm{mg}$ daily, aspirin $100 \mathrm{mg}$ daily, and was started on alendronate $70 \mathrm{mg}$ weekly. The CRP normalized within days of starting prednisone. At 1 month review she had gained 100 meters in exercise tolerance on level ground, and toe numbness had resolved. The popliteal and left pedal pulses became palpable. The prednisone dose was tapered by $10 \mathrm{mg}$ monthly. Statin therapy was not indicated.

A progress PET scan at 5 months showed significant improvement, with very mild residual uptake of the bilateral femoral and popliteal arteries (Figure 2). The progress CTA showed normal left lower-limb arteries with scattered short segments of mild stenoses of no hemodynamic significance; and persistent, although improved, segmental narrowing in the right superficial femoral and popliteal arteries; and right tibioperoneal trunk occlusion.

Personal non-commercial use only. The Journal of Rheumatology Copyright @ $@ 2010$. All rights reserved. 


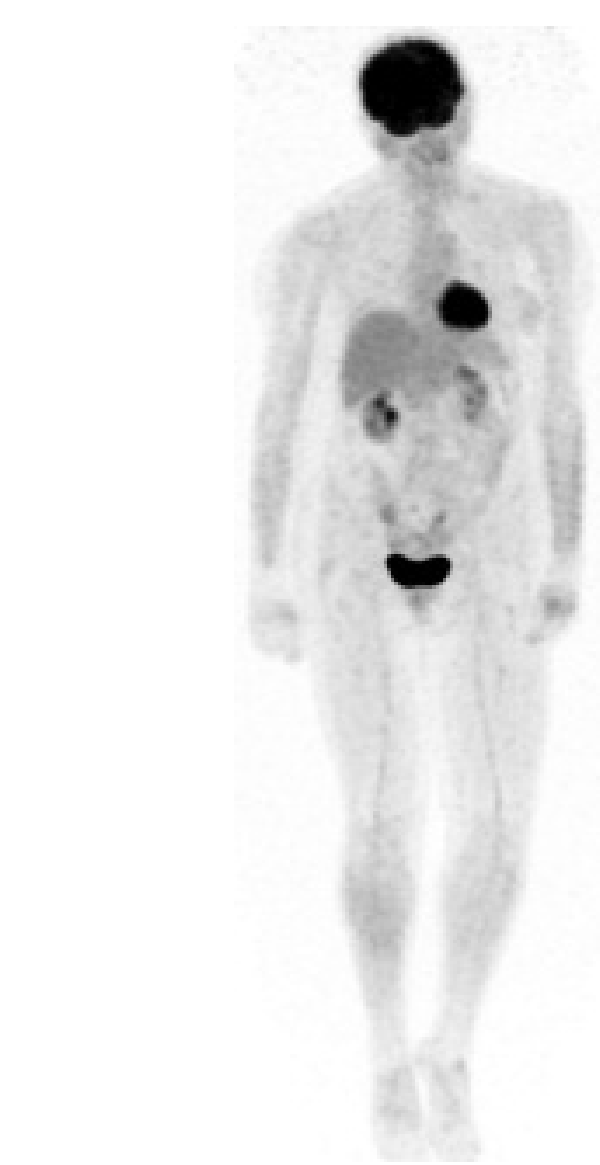

Figure 2. PET scan at 5 months post-treatment shows very mild uptake in the bilateral femoral and popliteal arteries, suggestive of marginal residual inflammation.

At 6 months, oral methotrexate was added as a steroid-sparing agent. After 14 months the right lower-limb claudicant symptoms were stable with claudication at 300 meters. She was receiving prednisone $12.5 \mathrm{mg}$ daily.

Although any artery may be affected by GCA, cranial vessel disease is the commonest site of disease ${ }^{1}$. Extracranial, large-vessel vasculitis is an uncommon presentation of GCA, occurring in 3\% to $15 \%$ of patients with $\mathrm{GCA}^{2}$. Thoracic aorta and its major branches are most commonly affect$\mathrm{ed}^{1-4}$, resulting in symptomatic upper-limb arterial disease. Extracranial vasculitis of the aorta, coronary, and peripheral limb arteries can cause significant morbidity and mortality, resulting in aortic dissection or rupture, myocardial infarcts, and limb amputations $s^{1,2,4,5}$.

Lower-limb GCA is rare, occurring in only $18 \%$ of extracranial GCA ${ }^{2}$, in which only a proportion will be symptomatic. As in our patient, lower-limb GCA shows a female predominance with a mean age of 52 years $^{6}$. It preferentially involves the femoropopliteal, deep femoral, and tibioperoneal arteries ${ }^{7}$.

Acute bilateral and rapidly progressive claudication, as in our patient, is the commonest presenting symptom of lower-limb $\mathrm{GCA}^{4,6,7}$. Other symptoms include peripheral paresthesias and Raynaud phenomenon ${ }^{1}$. Diagnosis may be delayed due to symptoms mimicking those of arteriosclerotic peripheral vascular disease $e^{4,7,8}$. Further, cranial signs and systemic inflammatory symptoms (fever, night sweats, anorexia, weight loss) may be very mild or absent in $\mathrm{GCA}^{1}$. Features atypical for arteriosclerotic peripheral vascular disease in our patient included her negligible atherosclerotic risk factors, the rapid weight loss, and progression of bilateral symptoms.
In our case GCA was confirmed on temporal biopsy, but less than 50\% of extracranial GCA cases have positive temporal artery biopsies ${ }^{1,2}$, and large vessels are difficult to biopsy. Therefore current diagnosis of LV-GCA relies on both the clinical scenario and noninvasive imaging such as duplex ultrasound, CTA, magnetic resonance imaging (MRI), and PET, as well as the often but not always elevated inflammatory markers.

Classic sonographic features of GCA on arterial duplex ultrasound include long, concentric, hypoechogenic thickening of the arterial wall ${ }^{7}$. Perivascular hypoechogenicity due to vessel wall edema, known as the "halo" sign, has a high specificity of $97 \%^{1,8}$, but a low sensitivity of $86 \%$ in the temporal artery ${ }^{8}$. Presence of complete occlusion has almost $100 \%$ specificity ${ }^{8}$. However, the mural thickening that persists throughout treatment correlates poorly with disease activity; and the halo sign in the temporal artery disappears after a mean of 16 days post-corticosteroid treatment ${ }^{9}$.

Contrast enhanced MRI/MR angiography is a useful diagnostic tool in LV-GCA ${ }^{10,11}$, demonstrating circumferential mural thickening of involved arteries with $81 \%$ sensitivity and $87 \%$ specificity ${ }^{1}$. MRI is valuable in detecting complications of LV-GCA such as aneurysms and stenoses ${ }^{1,11}$. However, a small prospective trial comparing PET and MRI in assessing LV-GCA disease activity showed higher nonspecific activity scores in MRI, possibly due to neoangiogenesis mimicking continued inflammatory disease $^{11}$.

Fluorine-18-deoxyglucose PET ( ${ }^{18} \mathrm{~F}$-FDG-PET) is an emerging investigation in large-vessel vasculitis. The level of FDG uptake correlates well with changing inflammatory activity, and is more inflammatory-specific than MRI ${ }^{11}$. As demonstrated in our case, the repeat CTA findings corresponded well with the progress PET findings. There is also complete anatomic agreement between duplex ultrasound and PET in identifying the distribution of large-vessel vasculitis ${ }^{8}$, with PET able to reveal additional sites of subclinical large-vessel disease in $80 \%$ of patients with $\mathrm{GCA}^{3}$. Of note, a prospective PET study did not show significant differences in uptake between 3 and 6 months of therapy, suggesting that persistent subclinical uptake post 3 months may not reflect true disease activity ${ }^{12}$. False-positives include active atherothrombotic disease or tissue repair ${ }^{11}$. Further, progress PET scans do not predict which patients are prone to relapse ${ }^{12}$.

The optimal treatment regimen for LV-GCA is undefined. Consensus management is extrapolated from cranial GCA: prompt high-dose corticosteroids tapered over 6-12 months, with prolonged therapy ranging from 1 to 5 years ${ }^{1}$. Persisting symptoms, as in our patient, often reflect fixed stenotic lesions in damaged arteries. As with classic temporal GCA, patients often relapse, requiring reescalation of the steroid dose ${ }^{6}$.

The ideal steroid-sparing agent in GCA is unknown, although methotrexate is used commonly despite conflicting data regarding its efficacy $^{1,3}$. In a metaanalysis of 3 studies, methotrexate $(10-15 \mathrm{mg} / \mathrm{wk})$ showed a modest reduction in the relapse rate and the cumulative dose of steroid therapy ${ }^{13}$

Low-dose aspirin, in conjunction with prednisone, reduces the risk of blindness or stroke in GCA by 5 -fold ${ }^{1,3}$. The role of antiplatelet agents in LV-GCA is undefined.

Two small studies failed to confirm the efficacy of statins in reducing ischemic complications or relapse rates in $\mathrm{GCA}^{14,15}$. However, benefits of statins in GCA cannot be excluded due to limitations of the studies ${ }^{14}$, and statin therapy should be considered in patients with GCA, given the increased cardiovascular risk of prolonged steroid treatment and the elderly patient population.

Anecdotal reports of balloon angioplasty for upper-limb LV-GCA have been favorable ${ }^{1}$. However, bypass, endarterectomy, and stenting of limb arteries often result in reocclusion, especially during the acute, uncontrolled inflammatory phase, and should be avoided unless acute ischemia threatens the limb $b^{6,9}$.

Reports on the role of exercise training in LV-GCA are scarce. However, vascular training is likely to be just as important an adjuvant therapy (especially for lower-limb GCA disease) as in lower-limb atherosclerotic peripheral vascular disease. 
Limited data suggest an equivalent, favorable prognosis between classical cranial GCA and LV-GCA ${ }^{1,9}$. However, the increased mortality in cases involving aortic aneurysms and dissections ${ }^{1}$ appears not to be modifiable by adequate and prolonged medical treatment, and early surgical intervention is recommended ${ }^{2}$.

The prognosis in lower-limb GCA is variable. A case report of 2-year followup angiography showed that marked stenosis in advanced lower-limb GCA arterial lesions remains fixed, but with new collateral flow, while less advanced stenosis in other arteries showed significant improvement ${ }^{16}$. Similarly, claudication ultimately regressed in $60 \%-70 \%$ of patients with lower-limb GCA on clinical followup despite a $17 \%-40 \%$ relapse rate ${ }^{6}$. Early case reports of LV-GCA describe limb amputations for severe ischemia refractory to corticosteroid therapy ${ }^{2,4}$, with $12.5 \%$ of cases of LV-GCA requiring limb amputations ${ }^{2}$. However, progression to advanced ischemia is rare overall ${ }^{4,9}$, especially with current use of high corticosteroid doses.

LV-GCA should be considered in middle-aged patients without significant atherosclerotic risk factors who present with claudicant symptoms, especially when bilateral and rapidly progressive. Cranial signs and systemic features of GCA may be very mild or absent. In conjunction with clinical assessment and other imaging modalities, PET may be a useful tool for accurate assessment and monitoring of disease distribution in lower-limb LV-GCA.

TED YI-ENG TSAI, MB, BS, BSci(Med), BA, Conjoint Associate Lecturer, University of New South Wales; DAVID MASSASSO, MB, BS, BSci(Med), FRACP, Rheumatology Staff Specialist, Liverpool Hospital, Sydney, Conjoint Associate Lecturer, University of New South Wales; PRANEAL SHARMA, MB, BS, FRANZCR, Senior Radiologist, Liverpool Hospital, Sydney; JOHN CROZIER, MB, BS, FRACS, DDU (Vasc), Vascular Surgeon, Liverpool Hospital, Senior Lecturer, University of NSW, Sydney, Australia. Address correspondence to Dr. T. Tsai; e-mail: t.tsai@unsw.edu.au

\section{REFERENCES}

1. Kawasaki A, Purvin V. Giant cell arteritis: an updated review. Acta Ophthalmol 2009;87:13-32.

2. Lie JT. Aortic and extracranial large vessel giant cell arteritis: a review of 72 cases with histopathologic documentation. Semin Arthritis Rheum 1995;24:422-31.

3. Hellmann DB. Giant cell arteritis and polymyalgia rheumatica. In: Imboden JB, Hellmann DB, Stone JH, editors. Current rheumatology diagnosis and treatment. 2nd ed. New York: McGraw-Hill; 2007.
4. Greene GM, Lain D, Sherwin RM, Wilson JE, McManus BM. Giant cell arteritis of the legs: clinical isolation of severe disease with gangrene and amputations. Am J Med 1986;81:727-33.

5. Finlayson R, Robinson JO. Giant-cell arteritis of the legs. BMJ $1955 ; 2: 1595-7$

6. Le Hello C, Levesque H, Jeanton M, Cailleux N, Galateau F, Peillon C, et al. Lower limb giant cell arteritis and temporal arteritis: followup of 8 cases. J Rheumatol 2001;28:1407-12.

7. Tato F, Hoffmann U. Clinical presentation and vascular imaging in giant cell arteritis of the femoropopliteal and tibioperoneal arteries. Analysis of four cases. J Vasc Surg 2006;44:176-82.

8. Schmidt WA, Blockmans D. Use of ultrasonography and positron emission tomography in the diagnosis and assessment of large-vessel vasculitis. Curr Opin Rheumatol 2005;17:9-15.

9. Schmidt WA, Moll A, Seifert A, Schicke B, Gromnica-Ihle E, Krause A. Prognosis of large-vessel giant cell arteritis. Rheumatology 2008;47:1406-8.

10. Bley TA, Warnatz K, Wieben O, Uhl M, Scholz C, Vaith P, et al. High-resolution MRI in giant cell arteritis with multiple inflammatory stenoses in both calves. Rheumatology 2005; 44:954-5.

11. Both M, Ahmadi-Simab K, Reuter M, Dourvos O, Fritzer E, Ullrich S, et al. MRI and FDG-PET in the assessment of inflammatory aortic arch syndrome in complicated courses of giant cell arteritis. Ann Rheum Dis 2008;67:1030-3.

12. Blockmans D, de Ceuninck L, Vanderschueren S, Knockaert D, Mortelmans L, Bobbaers H. Repetitive 18F-fluorodeoxyglucose positron emission tomography in giant cell arteritis: a prospective study of 35 patients. Arthritis Rheum 2006;55:131-7.

13. Mukhtyar C, Guillevin L, Cid MC, Dasgupta B, de Groot K, Gross $\mathrm{W}$, et al. EULAR recommendations for the management of large vessel vasculitis. Ann Rheum Dis 2009;68:318-23.

14. Garcia-Martinez A, Hernandez-Rodriguez J, Grau JM, Cid MC. Treatment with statins does not exhibit a clinically relevant corticosteroid-sparing effect in patients with giant cell arteritis. Arthritis Rheum 2004;51:674-8.

15. Narvaez J, Bernad B, Nolla JM, Valverde J. Statin therapy does not seem to benefit giant cell arteritis. Semin Arthritis Rheum 2007;36:322-7.

16. Garcia Vazquez JM, Carreira JM, Seoane C, Vidal JJ. Case report: superior and inferior limb ischaemia in giant cell arteritis: angiography follow up. Clin Rheumatol 1999;18:61-5.

J Rheumatol 2010;37:4; doi:10.3899/jrheum.090929 